\title{
Association between Alopecia Areata and Comorbid Allergies: Implications for Its Clinical Course
}

\author{
Hyun Ji Lee*, Nam-Soo Hong, ${ }^{1, *}$, Sang-Hyun Kim² ${ }^{2}$ Yong Hyun Jang \\ Departments of Dermatology, ${ }^{1}$ Preventive Medicine, and ${ }^{2}$ Pharmacology, School of Medicine, Kyungpook National University, Kyungpook \\ National University Hospital, Daegu, Korea
}

\section{Dear Editor:}

Few studies have reported that history of allergy and comorbid atopic dermatitis (AD) were associated with increased risk of alopecia areata (AA) and also with severe types of AA (i.e., alopecia totalis and alopecia universalis) ${ }^{1,2}$. Although there were no strong evidences in these studies with a small number of patients, AD has been clinically suspected as a poor prognostic factor of $\mathrm{AA}^{3}$. However, the influence of comorbid allergies in clinical outcomes of AA has not been reported previously. Thus, we attempted to find out a correlation between comorbid allergies and clinical course of AA. The protocol for the study was approved by the institutional review board of the Kyungpook National University Hospital (KNUH 201903-009).

Among total of 954 patients diagnosed with AA from 2011 to 2017 in Kyungpook National University Hospital, 664 patients who did not measure serum total immunoglobulin $\mathrm{E}$ (IgE), those with follow-up periods fewer than 6

Received October 29, 2019, Revised December 14, 2019, Accepted for publication December 17, 2019

*These authors have equally contributed to the article.

Corresponding author: Yong Hyun Jang, Department of Dermatology, School of Medicine, Kyungpook National University, 130 Dongdeok-ro, Jung-gu, Daegu 41944, Korea. Tel: 82-53-420-5838, Fax: 82-53-426-0770, E-mail: yhjang@knu.ac.kr

ORCID: https://orcid.org/0000-0003-1706-007X

Sang-Hyun Kim, Department of Pharmacology, School of Medicine, Kyungpook National University, 130 Dongdeok-ro, Jung-gu, Daegu 41944, Korea. Tel: 82-53-420-4838, Fax: 82-53-423-4838, E-mail: shkim72@knu.ac.kr ORCID: https://orcid.org/0000-0002-6160-7354

This is an Open Access article distributed under the terms of the Creative Commons Attribution Non-Commercial License (http://creativecommons. org/licenses/by-nc/4.0) which permits unrestricted non-commercial use, distribution, and reproduction in any medium, provided the original work is properly cited.

Copyright (c) The Korean Dermatological Association and The Korean Society for Investigative Dermatology months, and those with insufficient medical records were excluded. Finally, 290 patients included in this study. History of comorbid allergies (AD, allergic rhinitis, asthma) and disease progression were reviewed by medical records and photographs. Clinical courses compared between the patient's first and latest visit were evaluated into three grades, aggravation or no hair regrowth, partial hair regrowth, and complete hair regrowth. Mean treatment duration was 42.1 months. Main therapeutic modalities were topicals and intralesional steroid injection $(n=96)$, excimer laser and phototherapy $(n=36)$, systemic immunosuppressants $(n=43)$, diphenylcyclopropenone immunotherapy $(n=28)$ combined and/or intercurrent $(n=77)$, and others $(n=10)$. Statistical analysis used one-way ANOVA and chi-square test. There were 136 males and 154 females and mean duration of AA was 70.4 months. The mean age was $35.3 \pm 16.3$ years among total 290 patients, $77(26.6 \%)$ had comorbid allergies, $28(9.7 \%)$ with AD only, $26(9.0 \%)$ with allergic rhinitis only, $3(1.0 \%)$ for asthma only, and $20(6.9 \%)$ with more than two allergies (AD and allergic rhinitis, $\mathrm{n}=13 ; \mathrm{AD}$ and asthma, $\mathrm{n}=5$; allergic rhinitis and asthma, $n=2$ ). There was no statistical difference in the rate of patients with comorbid allergies according to the initial severity of AA (Fig. 1A). Then, we compared clinical courses of AA between with or without comorbid allergies, but it showed no statistical difference (Fig. 1B). In addition, there was no statistical difference of IgE levels according to the initial severity of AA (Fig. 1C). We divided four groups according to their IgE levels and compared the clinical courses, but there was also no significant difference (Fig. 1D). Table 1 summarized the complete hair regrowth rate in demographic and clinical characteristics. There were no statistically significant factors except for disease duration. However, 5 patients who had both comorbid allergies and very high IgE levels $(\geq 1,000$ 
A
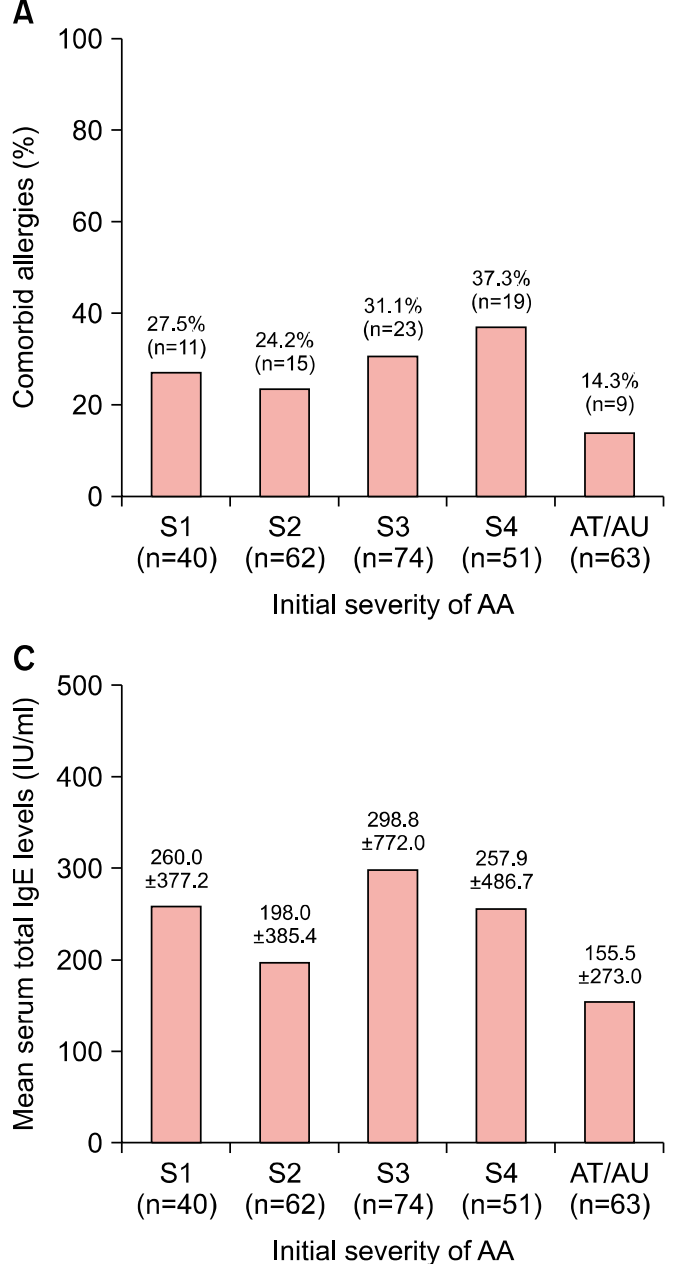

B
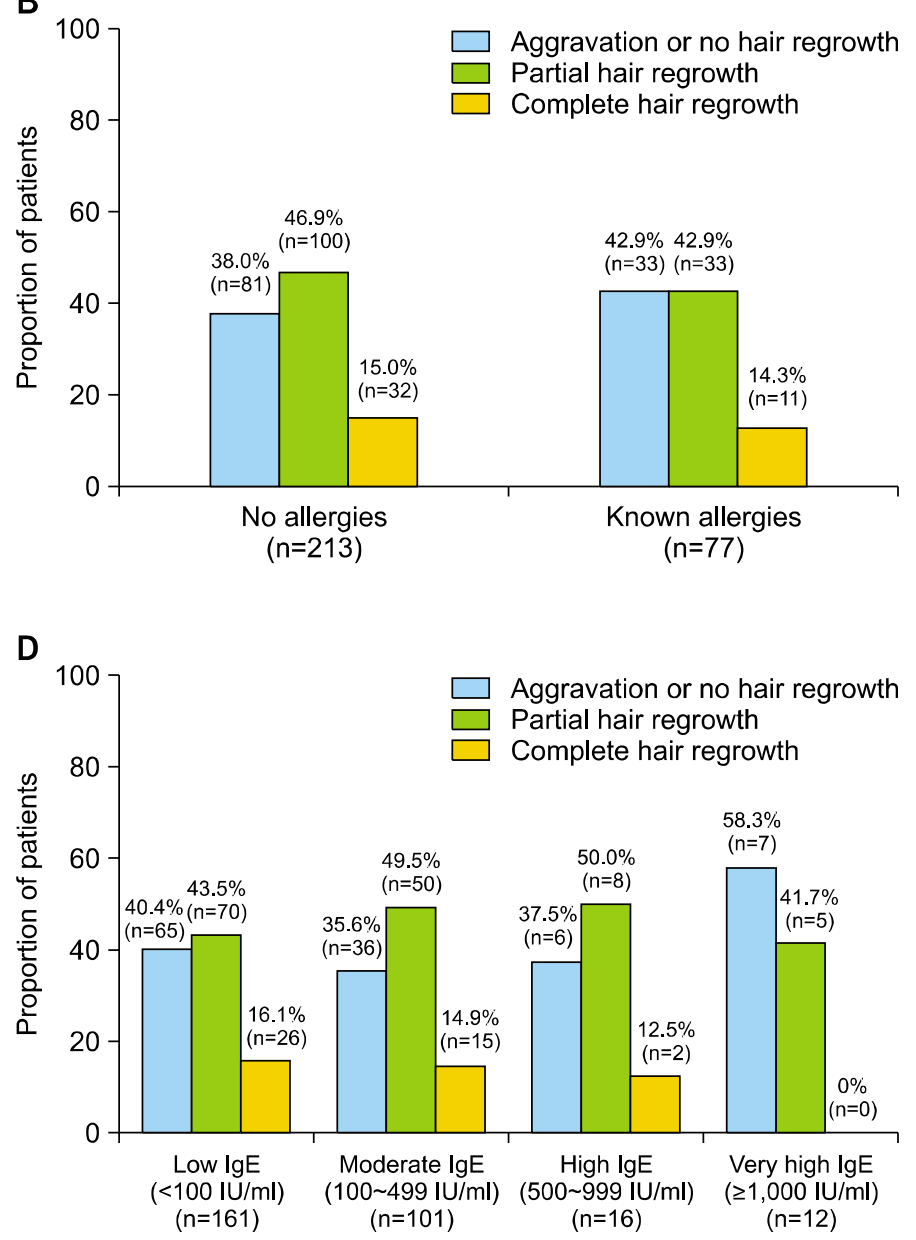

Fig. 1. The associations of the rate of patients with comorbid allergies and initial severity of $A A(A)$, clinical courses of $A A$ and comorbid allergies (B), mean serum total immunoglobulin E (IgE) levels and initial severity of AA (C), clinical courses of AA and serum total IgE levels (D) were investigated respectively, but none of them had statistically significant results. AA: alopecia areata, S0: no hair loss, S1: $<25 \%$ hair loss, S2: $25 \% \sim 49 \%$ hair loss, S3: 50\% 74\% hair loss, S4: $75 \% \sim 99 \%$ hair loss, AT: alopecia totalis, AU: alopecia universalis.

$\mathrm{IU} / \mathrm{ml})$, showed only aggravation or no hair regrowth. This presented the possibility of comorbid allergies and high IgE levels as poor prognostic factors of AA.

The immune response of AA has been traditionally explained to Th1 category. Although AD was known as Th2 category condition, it is now considered having complex heterogeneous immunopathogenesis involving Th1 and Th17 immune axis $^{4}$. So, both entities might have overlapping immunologic pathway theoretically. Unlike previous studies, this study investigated whether comorbid allergic diseases could affect the courses of AA. The main limitations of this study include its retrospective design, single-center site and a small number of patients showed complete hair regrowth which also resulted limitation of statistical analysis. In conclusion, there was no significant difference in the clinical courses of $\mathrm{AA}$ according to both comorbid allergic diseases and the level of serum total lgE.

\section{CONFLICTS OF INTEREST}

The authors have nothing to disclose.

\section{FUNDING SOURCE}

This research was supported by Basic Science Research Program through the National Research Foundation of Korea (NRF) funded by the Ministry of Education (NRF2018R1D1A3A03000686), and a grant of the Korea Health Technology R\&D Project through the Korea Health Industry Development Institute (KHIDI), funded by the Ministry of Health \& Welfare, Republic of Korea (grant number: HI18C0308). 
Table 1. Multivariable analyses of the effects of demographic and clinical characteristics on complete hair regrowth in patients with alopecia areata

\begin{tabular}{|c|c|c|c|}
\hline Characteristic & Number & $\begin{array}{l}\text { Complete hair } \\
\text { regrowth }\end{array}$ & $p$-value \\
\hline Sex & & & 0.7823 \\
\hline Male & 136 & $21(15.4)$ & \\
\hline Female & 154 & $22(14.3)$ & \\
\hline Age (yr) & & & 0.1063 \\
\hline $1 \sim 9$ & 30 & $3(10.0)$ & \\
\hline $10 \sim 19$ & 57 & $11(19.3)$ & \\
\hline $20 \sim 29$ & 45 & $11(24.4)$ & \\
\hline $30 \sim 39$ & 60 & $6(10.0)$ & \\
\hline $40 \sim 49$ & 56 & $4(7.1)$ & \\
\hline$\geq 50$ & 42 & $8(19.0)$ & \\
\hline Duration of AA (yr) & & & 0.0001 \\
\hline$<2$ & 85 & $25(29.4)$ & \\
\hline $2 \leq \mathrm{yr}<5$ & 99 & $10(10.1)$ & \\
\hline $5 \leq \mathrm{yr}<10$ & 61 & $4(6.6)$ & \\
\hline$\geq 10$ & 45 & $4(8.9)$ & \\
\hline Initial severity of $\mathrm{AA}$ & & & 0.3152 \\
\hline $\mathrm{S} 1$ & 40 & $9(22.5)$ & \\
\hline S2 & 62 & $10(16.1)$ & \\
\hline S3 & 74 & $10(13.5)$ & \\
\hline S4 & 51 & 9 (17.6) & \\
\hline $\mathrm{AT} / \mathrm{AU}$ & 63 & $5(7.9)$ & \\
\hline Comorbid allergies & & & 0.8759 \\
\hline Known allergies & 77 & $11(14.3)$ & \\
\hline No allergies & 213 & $32(15.0)$ & \\
\hline $\begin{array}{l}\text { Serum total IgE levels } \\
(\mathrm{IU} / \mathrm{ml})\end{array}$ & & & 0.4649 \\
\hline$<100$ & 161 & $26(16.1)$ & \\
\hline $100 \sim 499$ & 101 & 15 (14.9) & \\
\hline$\geq 500$ & 28 & $2(7.1)$ & \\
\hline \multicolumn{4}{|c|}{ Allergies×IgE levels (IU/ml) } \\
\hline \multicolumn{4}{|l|}{ No allergies } \\
\hline$<100$ & 121 & $20(16.5)$ & 0.5130 \\
\hline $100 \sim 499$ & 75 & $11(14.7)$ & \\
\hline$\geq 500$ & 17 & $1(5.9)$ & \\
\hline \multicolumn{4}{|l|}{ Known allergies } \\
\hline$<100$ & 40 & $6(15.0)$ & 1.000 \\
\hline $100 \sim 499$ & 26 & $4(15.4)$ & \\
\hline$\geq 500$ & 11 & $1(9.1)$ & \\
\hline Total & 290 & $43(14.8)$ & \\
\hline
\end{tabular}

Values are presented as number (\%). S0: no hair loss, S1: $<25 \%$ hair loss, S2: $25 \% \sim 49 \%$ hair loss, S3: $50 \% \sim 74 \%$ hair loss, S4: $75 \% \sim 99 \%$ hair loss, AT: alopecia totalis, AU: alopecia universalis, IgE: immunoglobulin $\mathrm{E}$.

\section{DATA SHARING STATEMENT}

Research data are not shared.

\section{ORCID}

Hyun Ji Lee, https://orcid.org/0000-0002-4222-1835

Nam-Soo Hong, https://orcid.org/0000-0002-6598-9126

Sang-Hyun Kim, https://orcid.org/0000-0002-6160-7354

Yong Hyun Jang, https://orcid.org/0000-0003-1706-007X

\section{REFERENCES}

1. Barahmani N, Schabath MB, Duvic M; National Alopecia Areata Registry. History of atopy or autoimmunity increases risk of alopecia areata. J Am Acad Dermatol 2009;61:581591.

2. Goh C, Finkel M, Christos PJ, Sinha AA. Profile of 513 patients with alopecia areata: associations of disease subtypes with atopy, autoimmune disease and positive family history. J Eur Acad Dermatol Venereol 2006;20:1055-1060.

3. Betz RC, Pforr J, Flaquer A, Redler S, Hanneken S, Eigelshoven $\mathrm{S}$, et al. Loss-of-function mutations in the filaggrin gene and alopecia areata: strong risk factor for a severe course of disease in patients comorbid for atopic disease. J Invest Dermatol 2007;127:2539-2543.

4. Brunner PM, Guttman-Yassky E, Leung DY. The immunology of atopic dermatitis and its reversibility with broad-spectrum and targeted therapies. J Allergy Clin Immunol 2017; 139(4S):S65-S76. 